\title{
Specific Heat of the Mixed Spin-1/2 and Spin-S Ising Model with a Rope Ladder Structure
}

\author{
J. KIŠŠOVÁ AND J. STREČKA
}

Department of Theoretical Physics and Astrophysics, Faculty of Science P.J. Šafárik University, Park Angelinum 9, 04001 Košice, Slovak Republic

The mixed spin-1/2 and spin- $S(S>1 / 2)$ Ising model on a rope ladder is examined by combining two exact analytical methods. By the decoration-iteration mapping transformation, this mixed-spin system is firstly transformed to a simple spin-1/2 Ising model on the two-leg ladder, which is then exactly solved by the standard transfer-matrix method. The thermal variations of the zero-field specific heat are discussed in particular.

PACS numbers: $05.50 .+\mathrm{q}, 75.40 . \mathrm{Cx}$

\section{Introduction}

One-dimensional Ising models are of fundamental scientific interest partly on behalf of their exact solubility [1] and partly due to the fact that they represent the simplest lattice-statistical models, which have found rich applications in seemingly diverse research areas [2]. In the present work, we shall provide the exact solution for the mixed spin-1/2 and spin- $S(S>1 / 2)$ Ising model on a rope ladder, which represents a rather novel magnetic structure emerging in several insulating bimetallic coordination compounds [3].

\section{Model and its exact solution}

Let us consider the spin-1/2 Ising ladder of $2 N$ sites with each of its horizontal and vertical bonds occupied by the decorating spin $S(S>1 / 2)$, as it is schematically shown in Fig. 1. For further convenience, the Hamiltonian of the model under investigation can be written as a sum of site Hamiltonians

$$
\mathcal{H}=\sum_{k=1}^{3 N} \mathcal{H}_{k}, \quad \mathcal{H}_{k}=-J S_{k}\left(\sigma_{k 1}+\sigma_{k 2}\right)-D S_{k}^{2}
$$

where each site Hamiltonian $\mathcal{H}_{k}$ involves all the interaction terms of one decorating spin. Above, $\sigma_{k \gamma}= \pm 1(\gamma=1,2)$ denote two vertex spins of the rope ladder 


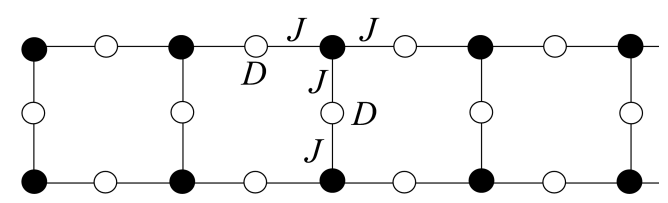

Fig. 1. A small fragment of the mixed-spin Ising model on the rope ladder. The full circles denote lattice positions of the vertex spins $\sigma_{j}= \pm 1$, while the empty ones label lattice positions of the decorating spins $S_{k}=-S,-S+1, \ldots,+S$.

that are nearest neighbours to the decorating spin $S_{k}=-S,-S+1, \ldots,+S$. The parameter $J$ labels pairwise exchange interaction between the nearest-neighbour spin pairs, while the parameter $D$ measures a strength of the single-ion anisotropy acting on the decorating spins.

The crucial step of our procedure lies in the calculation of the partition function, which can be firstly partially factorized to the expression

$$
\mathcal{Z}=\sum_{\left\{\sigma_{j}\right\}} \prod_{j=1}^{3 N} \sum_{n=-S}^{+S} \exp \left(\beta D n^{2}\right) \cosh \left(\beta J n\left(\sigma_{k 1}+\sigma_{k 2}\right)\right),
$$

where $\beta=1 /\left(k_{\mathrm{B}} T\right), k_{\mathrm{B}}$ is Boltzmann's constant, $T$ is the absolute temperature and the symbol $\sum_{\{\sigma\}}$ denotes the summation over all possible spin configurations of the vertex spins $\sigma_{j}$. Next, it is advisable to employ the generalised decoration-iteration mapping transformation [4]:

$$
\sum_{n=-S}^{+S} \exp \left(\beta D n^{2}\right) \cosh \left(\beta J n\left(\sigma_{k 1}+\sigma_{k 2}\right)\right)=A \exp \left(\beta R \sigma_{k 1} \sigma_{k 2}\right),
$$

which physically corresponds to removing all the interaction parameters associated with the decorating spins and replacing them by a new effective interaction $R$ between the remaining vertex spins. Since there are four possible spin states for each pair of vertex spins $\sigma_{k 1}$ and $\sigma_{k 2}$ and only two of them provide independent equations from the transformation formula (3), the mapping parameters $A$ and $R$ have to meet the following conditions:

$$
\begin{aligned}
& A^{2}=\left(\sum_{n=-S}^{+S} \exp \left(\beta D n^{2}\right) \cosh (2 \beta J n)\right)\left(\sum_{n=-S}^{+S} \exp \left(\beta D n^{2}\right)\right) \\
& \beta R=\frac{1}{2} \ln \left(\sum_{n=-S}^{+S} \exp \left(\beta D n^{2}\right) \cosh (2 \beta J n)\right)-\frac{1}{2} \ln \left(\sum_{n=-S}^{+S} \exp \left(\beta D n^{2}\right)\right) .
\end{aligned}
$$

By substituting the transformation (3) into Eq. (2) one gains the relation

$$
\mathcal{Z}(\beta, J, D)=A^{3 N} \mathcal{Z}_{0}(\beta, R),
$$

which connects the partition function $\mathcal{Z}$ of the mixed-spin Ising model on the rope ladder with the partition function $\mathcal{Z}_{0}$ of the simple spin-1/2 Ising model on two-leg ladder with the effective nearest-neighbour interaction $R$. It is worthwhile to remark that the partition function of the latter model can be rather easily calculated 
by applying the transfer-matrix method [1], which yields in the thermodynamic limit the following exact result:

$$
\mathcal{Z}_{0}=\left\{\cosh (3 \beta R)+\cosh (\beta R)+\sqrt{[\sinh (3 \beta R)-\sinh (\beta R)]^{2}+4}\right\}^{N} .
$$

Bearing this in mind, the exact solution for the partition function of the mixed-spin Ising model on the rope ladder is formally completed as it can be simply achieved by a mere substitution of the corresponding partition function (7) to the relationship (6), whereas both mapping parameters $A$ and $R$ have to be taken from Eqs. (4) and (5), respectively.

\section{Results and discussion}

Now, let us focus on thermal variations of the zero-field specific heat with the aim to shed light on how this dependence changes with the single-ion anisotropy. Before discussing the most interesting results, it should be mentioned that the zero-field specific heat can readily be obtained from the exact result for the partition function (6) with the help of basic thermodynamical-statistical relations. Even although the final expression is too cumbersome to write it down here explicitly, it is worthy noting nevertheless that it implies an independence on a sign of the exchange interaction $J$. In this respect, the temperature dependences of the zero-field specific heat discussed below remain in force regardless of whether ferromagnetic $(J>0)$ or ferrimagnetic $(J<0)$ spin system is considered.
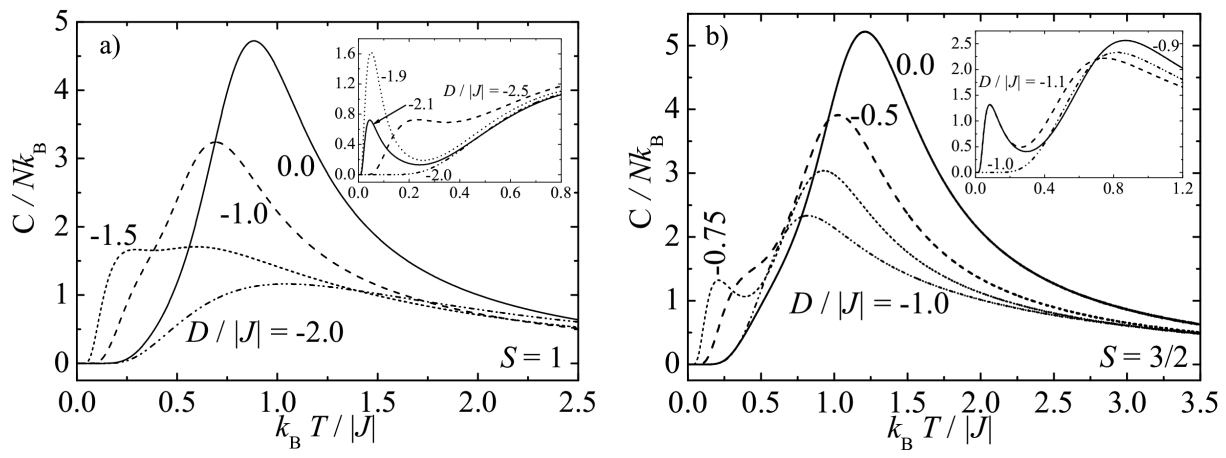

Fig. 2. The temperature dependences of zero-field specific heat for several single-ion anisotropies and two different spin values $S=1$ (a) and $S=3 / 2$ (b).

For illustration, Fig. 2a and b depict thermal variations of the zero-field specific heat for the rope ladder with the decorating spins $S=1$ and $S=3 / 2$. As one can see, the same general trends can be observed in both figures: the round maximum diminishes and shifts towards lower temperatures by decreasing the single-ion anisotropy. However, the most interesting temperature dependences can be found at sufficiently negative single-ion anisotropies close to $D / J=-2.0$ for the case with $S=1$ and respectively, $D / J=-1.0$ for the case with $S=$ 
$3 / 2$. In the vicinity of both these boundary values, the double-peak specific heat curves (low-temperature peaks are for clarity shown in the insets) appear due to the competition between two different spin configurations sufficiently close in energy. Actually, it can easily be verified that the general condition ensuring an energetic equivalence between two different spin states of decorating spins reads $D_{S \leftrightarrow S-1}=-2 J /(2 S-1)$. It should be also mentioned that the zero-field specific heat curves with a single maximum are recovered upon further decrease in the single-ion anisotropy. In conclusion, it seems valuable to extend our calculation to more general case with the non-zero magnetic field.

\section{Acknowledgments}

This work was supported by the Slovak Research and Development Agency under the contract No. LPP-0107-06 and the grant No. VEGA 1/2009/05.

\section{References}

[1] C.J. Thompson, Mathematical Statistical Mechanics, Princeton University Press, New Jersey 1979.

[2] C.J. Thompson, Classical Equilibrium Statistical Mechanics, Oxford University Press, New York 1988.

[3] M. Ohba, N. Maruono, H. Ōkawa, T. Enoki, J.-M. Latour, J. Am. Chem. Soc. 116, 11566 (1994); M. Ohba, N. Fukita, H. Ōkawa, J. Chem. Soc., Dalton Trans., 1733 (1997); M.L. Kahn, C. Mathonière, O. Kahn, Inorg. Chem. 38, 3692 (1999); H. Ōkawa, M. Ohba, Bull. Chem. Soc. Jpn. 75, 1191 (2002); M.S. El Fallah, J. Ribas, X. Solans, M. Font-Bardia, New J. Chem. 27, 895 (2003); Y.S. You, D. Kim, Y. Do, S.J. Oh, Ch.S. Hong, Inorg. Chem. 43, 6899 (2004).

[4] M.E. Fisher, Phys. Rev. 113, 969 (1959). 